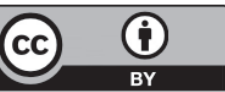

https://creativecommons.org/licenses/by/4.0/

\title{
ANÁLISIS SOBRE EL INCUMPLIMIENTO DE LA NORMATIVIDAD VIGENTE FRENTE A LA CADENA DE FRÍO EN LA RED DE DISTRIBUCIÓN DE LAS PYMES DEL SECTOR CÁRNICO DE BOVINOS EN BOGOTÁ*
}

\author{
Analysis on breach of regulatory from the cold chain \\ in the distribution network of SMEs sector in meat cattle Bogotá
}

MARÍA ALEXANDRA CARVAJAL URIBE**, LUBIAN DANILO URIBE ARBOLEDA***

Recibido: 24 de Abril de 2016. Aceptado: 27 de Mayo de 2016

DOI:http://dx.doi.org/10.21017/rimci.2016.v3.n6.a15

\begin{abstract}
Resumen
Se presentan los resultados de la investigación realizada en el marco del semillero de investigación "OPTIMIZACIÓN DE LA CADENA DE DISTRIBUCIÓN DEL CONGLOMERADO PYMES DEL SECTOR CÁRNICO DE BOVINOS DE BOGOTÁ D.C." , los cuales en el presente documento se enfocan únicamente a los hallazgos encontrados sobre el incumplimiento normativo que presentan algunas empresas pymes del sector cárnico de bovinos en Bogotá, frente al manejo de la cadena de frio.
\end{abstract}

Palabras clave: normatividad, PYMES, cadena de frío, distribución.

\begin{abstract}
Results of research conducted under the Research «Optimizing distribution chain conglomerate SMEs cattle meat industry Bogotá D.C.» which in this document focus only one the findings on the regulatory compliance of some companies in the meat sector SMEs cattle in Bogotá, regarding the management of the cold chain.
\end{abstract}

Keywords: regulations, SMEs, cold chain, distribution.

\section{INTRODUCCIÓN}

En este artículo se presentan los resultados derivados de la investigación descriptiva realizada a pequeñas y medianas empresas del sector cárnico de bovinos de Bogotá, enfatizando en el conocimiento que tienen las mismas sobre la normativi- dad vigente para Colombia sobre la cadena de frío para productos cárnicos, y se da una propuesta para su mejoramiento.

Para el desarrollo de la investigación se realizaron encuestas personalizadas a dieciséis empresas pymes del sector cárnico de bovinos de Bogotá.

* Resultado del trabajo de semillero «Optimización de la cadena de distribución del conglomerado PYMES del sector cárnico de bovinos de Bogotá D.C.» del grupo de investigación O.C.A liderado por el Ingeniero Guillermo Montes Paniza del programa de Ingeniería Industrial de la Corporación Universitaria Republicana.

* Estudiante de Ingeniería Industrial de la Corporación Universitaria Republicana. Correo electrónico: alexacaur@gmail.com

***Estudiante de Ingeniería Industrial de la Corporación Universitaria Republicana. Correo electrónico: 1duribe28@hotmail.com 
Aunado a lo anterior, se da una breve explicación de los términos usados para que el lector del presente artículo este familiarizado con los mismos.

\section{Marco teórico}

\section{A. Cadena de frío}

La revista Contexto Ganadero [1], a través de una entrevista realizada a funcionarios de FedegánFNG, enfatizó que para evitar cargas microbianas y la afectación de productos cárnicos es necesario mantener la cadena de frío desde el momento del sacrificio hasta el consumo entre 0 y 4 grados centígrados.

Pero, ¿Qué es la cadena de frío? Según Navarro en el documento logística en la cadena de frío de PROEXPORT Colombia, indica que la cadena de frío es aquel proceso en conjunto que garantiza la temperatura adecuada de los alimentos perecederos de acuerdo a sus características [2].

Su importancia radica en que cada año en Colombia y en el mundo aumentan las víctimas de intoxicación ${ }^{* * * *}$ por ingerir alimentos inocuos.

En otros términos, la cadena de frío es un proceso en el que cada pieza cumple un papel determinante para garantizar que la temperatura del alimento se mantenga en condiciones óptimas para su consumo.

PYMES, Según la Ley 590 del 10 de Julio del año 2000, se clasifican las empresas PYMES así:

1. Microempresa: Hasta 10 trabajadores. Activos inferiores a $501 \mathrm{smlv}$.

2. Pequeña Empresa: Entre 11 y 50 trabajadores. Activos mayores a 501 hasta $5.001 \mathrm{smlv}$

3. Mediana: Entre 51 y 200 trabajadores. Activos entre 5.001 y $15.000 \mathrm{smlv}$.

\section{B. Red de distribución}

Para Vatic Consulting Group, la red de distribución comprende desde la finalización de la producción hasta la entrega del producto al cliente, entendiendo que los clientes no necesariamente son los consumidores finales de los productos, sino incluso pueden ser el inicio de una nueva cadena [3].

Por su parte Chopra \& Meindl consideran que la cadena está conformada por todas las partes que hacen parte del proceso directa o indirectamente pasando por el proveedor, el transporte, los vendedores e incluso los clientes, cuyo objetivo es maximizar el valor agregado [4].

1. Elementos de la cadena de distribución:

La cadena de distribución está conformada por: diseño de la red de distribución, almacén, manejo de inventarios y portafolio de productos, gestión del transporte, servicio al cliente, indicadores de gestión [5].

2. Diseño de la red de distribución

Chopra \& Meindl manifiestan que para tomar decisiones acertadas frente al diseño de la red de distribución se deben tener en cuenta aspectos como las instalaciones, la ubicación de las mismas y su capacidad instalada, así como saber a qué mercados están dirigidos, lo cual generará un impacto positivo en el desempeño [6]. De dichas decisiones depende la permanencia y el éxito de las empresas, una mala decisión en la ubicación impactaría en los costos de traslados e incluso llevaría al cierre de algunas, por lo tanto es una decisión que debe evaluarse a largo plazo.

Por otra parte, al realizar fusiones o alianzas estratégicas con otras empresas, las decisiones frente al diseño de la red deben ser evaluadas conforme la empresa crece, es decir, deben evaluarse nuevas ubicaciones o ampliaciones de las instala-

**** Según datos de la Organización Mundial de la Salud (OMS), cerca de dos millones de personas mueren anualmente por consumir alimentos de forma insegura, en su mayoría en países en desarrollo. 
ciones para cubrir la demanda y tener una capacidad de respuesta efectiva.

Como afirma Chopra y Meindl existe un gran número de factores que influyen en las decisiones del diseño, entre los que encontramos factores, tecnológicos, económicos, políticos, estratégicos entre otros [4].

\section{Almacén}

«Consiste en la actividad que tiene como objetivo realizar la gestión de inventarios, conservación, manipulación y almacenamiento de bienes de consumo y medios de producción, diseño de almacenes y la explotación de los medios técnicos utilizados, equipos de manipulación y medios de almacenamiento y medición» [7].

La importancia del almacén radica en que permite tanto la conservación como la manipulación de los productos de manera correcta, asegurando el suministro ininterrumpido de los mismos evitando así que se vean afectadas otras áreas o el servicio como tal.

Ferrín establece algunos beneficios de la gestión de stock de almacenes, dentro de los que encontramos [8]:

Rotación: En este punto contempla la renovación del producto, en otras palabras, el flujo que tenga el producto respecto a la cantidad en existencia.

El nivel de la rotación depende de si el producto es perecedero o no e incluso de la obsolescencia del mismo.

El Margen: Este se obtiene de la diferencia entre las ventas y el costo de las mismas.

La Rentabilidad del Stock: El equilibrio entre rotación y el margen da como resultado el fin mismo de la gestión de stocks que es mejorar la rentabilidad.

\section{Inventarios}

Gutiérrez y Vidalb, concluyeron que esta actividad (transversal en la red de distribución) es por demás un aspecto complejo dentro del sector eco- nómico por cuanto la inversión en el mantenimiento y gestión de los inventarios de materia prima, producto en proceso o producto terminado son elevados [9]. Aunado a lo anterior, en este punto es claro que para que las empresas pymes del sector cárnico sean competitivas en un mercado marcado por el libre comercio se tendrán que definir unos métodos y unas políticas de control de los inventarios a lo largo de toda la cadena, y así evitar gastos por encima de lo necesario.

\section{E. Portafolio de productos}

La clasificación del portafolio de productos se puede realizar en dos partes:

Make To Stock (MTS: Hacer para almacenar) «En este sistema se fija el nivel de inventario deseado y se produce lo necesario para mantenerlo» [10].

Make To Order (MTO: hacer bajo pedido). «No hay inventario de producto terminado y simplemente se produce lo que la demanda ha solicitado, si alcanza la capacidad instalada» [10]

Cada empresa de acuerdo a sus políticas y necesidades de servicio podrá definir qué sistema de portafolio es más conveniente.

\section{F. Transporte}

«El transporte se ocupa de todas las actividades relacionadas directa e indirectamente con la necesidad de situar los productos en los puntos de destino correspondiente, de acuerdo con unas condiciones de seguridad, servicio, costo» [11]. Al escoger un modelo de distribución se debe contemplar previamente el medio de transporte que se utilizará, para esta decisión y la escogencia del vehículo como tal se deben tener en cuenta factores como:

1. Distancia de los clientes.

2. Tipo de producto a transportar (perecedero/no perecedero, volumen, grado de peligrosidad, peso).

3. Legislación de acuerdo a cada país.

4. Costos de transporte en caso de que sea propio o contratado. 
a. Transporte de Carne

En Colombia, el Ministerio de Transporte a través de la Resolución 002505 de 06 de septiembre de 2004, estableció las condiciones que deben cumplir los vehículos para el transporte de carne, pescado o alimentos fácilmente corruptibles.

\section{G. Servicio al Cliente}

Se debe tener claro que sin clientes ninguna empresa sobreviviría, sin embargo muchas veces se cae en el error de pensar que el servicio al cliente es responsabilidad exclusiva de las personas que tienen contacto directo con el cliente, como vendedores o impulsadoras, sin embargo dicha idea esta mandada a recoger y con la puesta en marcha de la idea del mejoramiento continuo el servicio al cliente recae en toda empresa y sus trabajadores, quienes garantizan que el producto o servicio sea entregado al cliente con la calidad, atención, y oportunidad requeridas para alcanzar su satisfacción.

Beltrán, Rivas y Muñuzuri coinciden que la finalidad del servicio al cliente es asegurarse que la empresa tiene la capacidad para dar respuesta a las demandas de sus clientes en las condiciones especificadas y adicionalmente realizar un seguimiento al mismo [12].

\section{H. Indicadores de Gestión}

Los indicadores de gestión constituyen una fuente de información primordial, en la que se pueden detectar las condiciones reales en las cuales se encuentra la empresa, en cuanto a aspectos como la calidad, la eficiencia y la eficacia, la productividad entre otras, y por lo tanto le permite medir su desempeño (D.O.F.A) ${ }^{* * * *}$ para así tomar decisiones encaminadas al mejoramiento de la misma.

«Todo se puede medir y por tanto se puede controlar, por lo tanto lo que no se mide no se puede controlar» [13].

\section{Normatividad vigente para Colombia}

A continuación se presenta algunos Decretos, Circulares y Resoluciones vigentes para Colombia ateniente a la cadena cárnica (se toma literalmente de la normatividad) [14, 15]:

\section{FEDEGAN}

\section{a. Decreto 1500 de 2007}

Por la cual se establece el reglamento técnico a través del cual se crea el Sistema Oficial de Inspección, Vigilancia y Control de Carne, Productos Cárnicos Comestibles y derivados Cárnicos destinados para el consumo humano.

\section{b. Resolución 072 de 2007}

Por la cual se establece el manual de buenas prácticas de manejo para la producción y obtención de piel de ganado bovino y bufalino.

\section{c. Resolución 2905 de 2007}

Por la cual se establece el reglamento técnico sobre los requisitos sanitarios y de inocuidad de la carne y productos cárnicos comestibles de las especies bovinas y bufalinas destinados para el consumo humano.

\section{d. Resolución 18119 de 2007}

Por la cual se reglamentan los requisitos del Plan Gradual de Cumplimiento para las plantas de beneficio y desposte de bovinos y bufalinos.

e. Decreto 2278 de 1982

Por la cual se reglamenta parcialmente el título V de la Ley 09 de 1979 en cuanto al sacrificio de animales de abasto público o para consumo humano y el procesamiento, transporte y comercialización de su carne.

*ork\% Herramienta analítica para determinar Debilidades, Oportunidades, Fortalezas y Amenazas. recuperado el 17 febrero de 2015 de http:// www.degerencia.com/tema/analisis_dofa 


\section{INVIMA}

a. Circular Externa Conjunta 016 de 2015

Implementación del sistema oficial de inspección, vigilancia y control de la carne y productos cárnicos comestibles para plantas de beneficio animal...

b. Resolución 240 de 2013

... expendio, transporte, importación o exportación de carne y productos cárnicos comestibles. ...

c. Resolución 242 de 2013

... o exportación de carne y productos cárnicos comestibles. ...

d. Resolución 3753 de 2013

Por la cual se definen los lineamientos técnicos para la formulación de planes de acción de inspección, vigilancia y control de la carne y productos cárnicos comestibles a lo largo de la cadena y se dictan...

e. Decreto 1500 de 2007

Por el cual se establece el reglamento técnico a través del cual se crea el Sistema Oficial de Inspección, Vigilancia y Control de la Carne, productos cárnicos comestibles y derivados carnicos destinados...

f. Resolución 14985 de 1998

Por la cual se autoriza el uso de unas enzimas y de la Primaricina en productos cárnicos. ...

\section{g. Decreto 2131 de 1997}

Por el cual se dictan disposiciones sobre productos cárnicos procesados. ...

h. Decreto No 216201 AGO de 1983

Por el cual se reglamenta parcialmente el título V de la ley 09 de 1979, en cuanto a producción, procesamiento, transporte y expendio de los productos cárnicos procesados...

i. Resolución 3753 del 24 de septiembre de 2013

Por la cual se definen los lineamientos técnicos para la formulación de planes de acción de inspección, vigilancia y control de la carne y productos cárnicos comestibles a lo largo de la cadena y se dictan...

j. Resolución 242 del 31 de enero de 2013

... cárnicos comestibles, el siguiente texto: Deroga la resolución 4287 de 2007 y las disposiciones que le sean contrarias. El artículo 58 de esta resolución fue derogado por la resolución 3753 del 24 de septiembre...

k. Resolución 240 del 31 de enero de 2013

... importación o exportación de carne y productos cárnicos comestibles, el siguiente texto: Indicar que deroga las resoluciones No 2905 y 4282 de 2007 y las disposiciones que le sean contrarias. El artículo...

\section{Metodología}

A través de un cuestionario, se indagaron a 16 empresas PYMES del sector cárnico de bovinos de la ciudad de Bogotá sobre su conocimiento sobre los documentos exigidos en Colombia, para evitar la ruptura de la cadena de frío.

La encuesta fue diseñada previamente dentro del proceso de investigación y estuvo sometida a varias modificaciones del grupo investigador, así como a la respectiva revisión del docente investigador Ing. Guillermo Montes Paniza.

Para este caso el tipo de investigación utilizada fue Exploratoria, se utilizó para identificar la problemática.

\section{Resultados}

Se presentan resultados de las encuestas realizadas a la empresa PYMES del sector cárnico de 
bovinos de la ciudad de Bogotá, cuyos datos son de naturaleza netamente cualitativa, específicamente a las preguntas 17, 18 y 19.

A. ¿Cuenta con tecnología dirigida a la conservación de los productos? Tabla 1.

B. ¿Respecto a la normatividad, sabe usted cuales son los documentos exigidos en Colombia, para evitar la ruptura de la cadena de frío? (¿se cumplen?) Tabla 2.

C. ¿Con que tipo de capacitación o certificación cuenta el personal, frente la red de distribución? Tabla 3.

La tecnología dirigida a la conservación de los alimentos con que cuenta el 100\% de las empresas es la refrigeración, ya que todas tienen cuarto frío y/o cuarto de refrigeración y/o neveras; de ellos un $37.5 \%$ dijo contar con cuarto frío, un $12,5 \%$ cuenta con ambos; un $12.5 \%$ con cuarto de congelación; un $12,5 \%$ cuenta con cuarto de refrigeración cuarto frío, cuarto de congelación y nevera; otro tanto cuenta con un cuarto de refrigeración, un cuarto de congelación y neveras; un $12,5 \%$ dispone de cuarto de refrigeración y neveras.

Un $68,75 \%$ de las empresas dijo conocer la normatividad, documentos exigidos para evitar la ruptura de la cadena del frío, mientras que el 31,25\% de las empresas dijo no conocer la normatividad, documentos exigidos para evitar la ruptura de la cadena del frío. De los que dijeron conocer y aplicar la normatividad un $12,5 \%$ dijo que es la administración quien la realiza (maneja, hace seguimiento); otro tanto dijo únicamente sí. Un 6,25\% dijo conocerla pero que no se cumple; otro tanto dijo conocer del taller, plan de saneamiento, alimentos y manejo; un mismo porcentaje dijo que se debe realizar rigor mortis para despostar; igual porcentaje dijo que tanto en el sacrificio como en la venta; el mismo porcentaje dijo realizarle seguimiento a los trabajadores para que se cumpla tanto del Mi-

Tabla 1. ¿Cuenta con tecnología dirigida a la conservación de los productos?

\begin{tabular}{|c|c|c|}
\hline No. & Empresa & ¿Cuenta con tecnología dirigida a la conservación de los productos? \\
\hline 1 & Carnes 20 de julio & $\begin{array}{l}1 \text { cuarto frío, } 1 \text { cuarto de congelación }-5 \text { y }-9^{\circ} \mathrm{C} \text {, agrupados por canastas, } \\
1 \text { nevera. }\end{array}$ \\
\hline 2 & Carnes Puerto Madero & Cuarto frío para conservación de las carnes, no informa temperatura. \\
\hline 3 & Cárnicos Torino & Cuenta con 3 cuartos fríos no informan temperatura. \\
\hline 4 & Carulla. Calle 63. & $\begin{array}{l}\text { Atmósfera modificada, es decir que el sistema extrae el oxígeno de la bandeja } \\
\text { manteniendo la vida útil de la carne, también tenemos empaques de última } \\
\text { generación los cuales prolongan las condiciones de los productos. }\end{array}$ \\
\hline 5 & Distribuidora Maxi Cárnicos Lupe & Sí, congeladores, temperaturas. \\
\hline 6 & La Fortaleza & 1 Cuarto congelación, $1\left(-4^{\circ} \mathrm{C}\right)$ cuarto de refrigeración $\left(-2^{\circ} \mathrm{C}\right) 5$ neveras. \\
\hline 7 & Coop. de carnes La Mejor del Llano & $\begin{array}{l}\text { Tenemos un cuarto frío, y tres neveras, dos de refrigeración }\left(-4,0 \mathrm{~g}^{\circ} \mathrm{C} \text { y }-9,4 \mathrm{~g}^{\circ} \mathrm{C}\right) \\
\text { y una de congelación a }-17 \mathrm{~g}^{\circ} \mathrm{C} \text {. }\end{array}$ \\
\hline 8 & Carnes Frías Las Palmas & $\begin{array}{l}2 \text { cuartos abajo y } 2 \text { arriba, } 2 \text { neveras congelación, refrigeración igual para } \\
\text { ambas neveras }-2,7 \mathrm{~g}^{\circ} \mathrm{C} \text {. }\end{array}$ \\
\hline 9 & HB Carnes P C SAS & Congelación y refrigeración $\left(-4 \mathrm{~g}^{\circ} \mathrm{c}-20 \mathrm{~g}^{\circ} \mathrm{c}\right)$ y neveras de 1 a $\left.0^{\circ} \mathrm{C}\right)$. \\
\hline 10 & La Número Uno & 1 cuarto de congelación y 1 cuarto de refrigeración y una nevera a $4^{\circ} \mathrm{C}$. \\
\hline 11 & Carnes el Cuadril & $\begin{array}{l}\text { Cuarto de refrigeración entre } 0 \text { y } 4^{\circ} \mathrm{C} \text {, cuarto de congelación entre }-1 \text { y }-10^{\circ} \mathrm{C} \\
\text { y } 2 \text { neveras a } 2^{\circ} \mathrm{C} \text {. }\end{array}$ \\
\hline 12 & Solo Postas & Cuarto frío para el control de temperaturas. \\
\hline 13 & Carnes Los Andes & $\begin{array}{l}\text { El carro llega de los frigoríficos San Martín y de Santander y aquí lo despostan } \\
\text { y luego lo envían al cuarto frío. }\end{array}$ \\
\hline 14 & Carnes San Juan & 1 cuarto de refrigeración $\left(-5 \mathrm{~g}^{\circ} \mathrm{C}\right) 2$ neveras $\left(-4\right.$ y $\left.-41 / 2 \mathrm{~g}^{\circ} \mathrm{C}\right)$. \\
\hline 15 & Carnes San Julián & 1 cuarto de refrigeración a $-2^{\circ}$ y dos neveras de acuerdo a la cantidad de carne. \\
\hline 16 & Frigo Carnes La 19 & $\begin{array}{l}\text { Sí, y dependiendo de la carne que se almacene así mismo manejan la temperatura } \\
\text { en el cuarto de frío. }\end{array}$ \\
\hline
\end{tabular}


Tabla 2. ¿Respecto a la normatividad, sabe usted cuáles son los documentos exigidos en Colombia, para evitar la ruptura de la cadena de frío? [¿se cumplen?]

\begin{tabular}{lll}
\hline No. & Empresa & $\begin{array}{c}\text { ¿Respecto a la normatividad, sabe usted cuáles son los documentos exigidos en } \\
\text { Colombia, para evitar la ruptura de la cadena de frío? (¿se cumplen?) }\end{array}$ \\
\hline 1 & Carnes 20 de julio & No conocemos. \\
2 & Carnes Puerto Madero & Si, se conoce y la cumplen, realizan seguimientos a los empleados para hacer \\
& & cumplir con la reglamentación exigida por el frigorífico y por el ministerio. \\
3 & Cárnicos Torino & Si. \\
4 & Carulla. Calle 63. & Por supuesto, somos delaspocs empresas del país con certificado ICONTEC, HACCP. \\
& & Pata la producción y empaque de carne bovina, se mantiene todo a cero ${ }^{\circ}$ C. \\
5 & Distribuidora Maxi Cárnicos Lupe & Taller, plan de saneamiento, alimentos y manejo. \\
6 & La Fortaleza & No. \\
7 & Coop. de carnes La Mejor del Llano & No conozco la normatividad. \\
8 & Carnes Frías Las Palmas & No. \\
9 & HB Carnes P C SAS & Si. Tanto como en el sacrificio como la venta al usuario final. \\
10 & La Número Uno & No, pero sabe que existe. \\
11 & Carnes el Cuadril & Sí, se debe realizar rigor mortis para despostar y se vende. \\
12 & Solo Postas & Si. \\
13 & Carnes Los Andes & Si, se implementan a cada uno que se establece. \\
14 & Carnes San Juan & Síla administración hace seguimiento para que se cumplan pero no se sabe que \\
& & sucede después de la venta. \\
15 & Carnes San Julián & Sí, pero no se cumple porque desde el Frigorífico no lo tienen en cuenta. \\
16 & Frigo Carnes La 19 & Sí, la maneja la administración. \\
\hline
\end{tabular}

Tabla 3. ¿Con qué tipo de capacitación o certificación cuenta el personal, frente la red de distribución?

\begin{tabular}{|c|c|c|}
\hline No. & Empresa & $\begin{array}{l}\text { ¿Con qué tipo de capacitación o certificación cuenta el personal, frente a la red } \\
\text { de distribución? }\end{array}$ \\
\hline 1 & Carnes 20 de julio & Carnet, manejo de alimentos - matadero ayuda a certificar al personal. \\
\hline 2 & Carnes Puerto Madero & Curso de Manipulación de alimentos y curso básico de saneamiento. \\
\hline 3 & Cárnicos Torino & Curso de Manipulación de alimentos y curso básico de saneamiento. \\
\hline 4 & Carulla. Calle 63. & $\begin{array}{l}\text { Todo el personal está altamente calificado para asegurar la calidad, antes del } \\
\text { ingreso reciben capacitación y durante su permanencia en la empresa se brinda } \\
\text { periódicamente entrenamiento en nueva tecnología, y se evita que cualquier } \\
\text { agente externo pueda contaminar. }\end{array}$ \\
\hline 5 & Distribuidora Maxi Cárnicos Lupe & Normas de higiene. \\
\hline 6 & La Fortaleza & $\begin{array}{l}\text { Experiencia, capacitación interna es decir sabemos que debemos cumplir con } \\
\text { las normas como el uso de guantes, sin necesidad de recibir capacitación de eso. }\end{array}$ \\
\hline 7 & Coop. de carnes La Mejor del Llano & $\begin{array}{l}\text { Para el ingreso se exige capacitación en cortes, debemos usar gorro y guantes } \\
\text { y contamos con afiliación a la ARL. }\end{array}$ \\
\hline 8 & Carnes Frías Las Palmas & Carnet de manipulación. Guantes, ARL, EPS. \\
\hline 9 & HB Carnes P C SAS & $\begin{array}{l}\text { Se contrata basados en la experiencia, están afiliados a todo lo de ley. Carnet de } \\
\text { manipulación. }\end{array}$ \\
\hline 10 & La Número Uno & Experiencia y certificación en manejo de alimentos (colabora la empresa). \\
\hline 11 & Carnes el Cuadril & Curso de manipulación de alimentos y ARL. \\
\hline 12 & Solo Postas & Curso de manipulación de alimentos y curso básico de saneamiento. \\
\hline 13 & Carnes Los Andes & Manifiesta que se maneja más por práctica y experiencia. \\
\hline 14 & Carnes San Juan & $\begin{array}{l}\text { Curso de manipulación de alimentos y capacitación por parte de la empresa } \\
\text { para manejo del producto. }\end{array}$ \\
\hline 15 & Carnes San Julián & $\begin{array}{l}\text { Curso de manipulación de alimentos, utiliza el guante de seguridad y tapabocas, } \\
\text { protectores de brazo, ARL. }\end{array}$ \\
\hline 16 & Frigo Carnes La 19 & $\begin{array}{l}\text { Sí, tienen Ingeniería de alimentos que capacita en el manejo y manipulación } \\
\text { del producto. }\end{array}$ \\
\hline
\end{tabular}


nisterio como por el Frigorífico San Martín; el mismo porcentaje dijo que se implementa a cada uno; por último un 6,25\% (una empresa) dijo ser de las pocas empresas con certificados ICONTEC y HACCAP para la producción y empaque de carne bovina.

El 68,75\% de las empresas dijo tener su personal capacitado en manipulación de alimentos; un $12,5 \%$ dijo exigir al ingreso a sus trabajadores práctica y experiencia, de los cuales el $50 \%$ capacita interna; un 6,25\% dijo exigir capacitación en corte de carnes a sus trabajadores; otro tanto dijo normas de higiene; igual porcentaje dijo capacitar su personal antes del ingreso y durante la permanencia en la empresa.

\section{Propuesta}

No se trata solo de conocer la normatividad, se trata de que las empresas PYMES del sector cárnico de bovinos de la ciudad de Bogotá se apropien de la importancia que tiene el correcto manejo de la cadena de frío dentro de la red de distribución y la apliquen en sus empresas.

La capacitación a todos los actores de la cadena de frío (incluyendo a los consumidores) es de gran importancia ya que esto minimizaría las intoxicaciones [16], por lo tanto nuestra propuesta está enfocada principalmente a:

1. Realizar capacitaciones junto con profesionales expertos en el tema, para superar los vacíos legales que existen frente al control de la cadena de frío especialmente en la red de distribución, lo anterior basados en la normatividad vigente para Colombia, principalmente en la Resolución 2905 de 2007 de FEDEGÁN en su Artículo 15 «CAPACITACIÓN» [14].

2. Documentar a los supervisores para que ejerzan mejor control de la temperatura de sus productos.

3. Mantener indicadores de tiempo-temperatura en los furgones, contenedores y neveras durante toda la red de distribución (incluso si no se cuenta con vehículos propios)
4. Finalmente, que los consumidores reciban información (volantes-cartillas) previamente a su compra acerca de la forma correcta de manipular el producto.

\section{ConCLUSIÓN}

Como resultado de la investigación realizada, se puede concluir que, si bien es cierto todas las empresas encuestadas cuentan con cuartos fríos para la conservación de los alimentos, no se tiene claro a que temperatura deben estar sin que afecte la calidad del producto, adicionalmente, muchas de las empresas encuestadas no conocen la normatividad vigente para Colombia para mantener la cadena de frío, y algunas que dicen conocerla no la tienen en cuenta o la dejan solo a responsabilidad de los administradores, finalmente, y respecto a la capacitación estas están enfocadas principalmente a la manipulación de alimentos de sus empleados, sin embargo ninguna de ellas manifestó tener en cuenta el conocimiento previo que tienen sus clientes para evitar la ruptura de la cadena de frío.

Por lo anterior, los esfuerzos de las empresas pymes del sector cárnico de bovinos de Bogotá, que busquen ofrecer a sus clientes productos de alta calidad, aumentando así su fidelización y confianza y disminuir sus pérdidas económicas por deterioros en los productos, se debe centrar en la capacitación sobre el control de la cadena de frío a lo largo de toda la red de distribución.

\section{REFERENCIAS}

[1] Federación Colombiana de Ganaderos, «Cadena de frío en cárnicos, un proceso que no se debe interrumpir,» Contexto Ganadero, 2014.

[2] L. Navarro, «Logística en la Cadena de Frío.» PROEXPORT COLOMBIA, 2015.

[3] Vatic Consulting Group. (s.f.), «Cadena de Suministro».

[4] S. Chopra \& P. Meindl, Administración de la cadena de suministro. Estrategia, planeación y operación., Tercera edición. México: Pearson Educación, 2008.

[5] S. Crone, «Logística: Definición, Distribution and logistics», 2012.

Rev. Ingeniería, Matemáticas y Ciencias de la Información Vol. 3 / Núm. 6 / julio - diciembre de 2016; pág. 59-67 
[6] S. Chopra S. \& P. Meindl, Administración de la cadena de suministro. Estrategia, planeación y operación., Tercera edición. México: Pearson Educación, 2008.

[7] L. Muñoz, Libro de logística de almacenes. Cuba: LR Muñoz, 2009.

[8] A. Ferrín, Gestión de stocks en la logística de almacenes. FC Editorial, 2007.

[9] V. Gutiérrez \& C. Vidalb, «Modelos de Gestión de Inventarios en Cadenas de Abastecimiento: Revisión de la Literatura Inventory Management Models in Supply Chains.» A Literature Review, 2008.

[10] G. Lavalle \& E. Santucci, «Flexibilidad productiva en un sistema mixto de fabricación a pedido y para inventario,» Revista de instituciones, ideas y mercados, pp. 37-46, 2009.

[11] J. Tejero, Logística integral: la gestión operativa de la empresa. ESIC editorial, 2007.

[12] J. Beltrán, M. Rivas, J. Muñuzuri, «Sistemas de gestión logística: un enfoque para la evaluación, integración y mejora de los procesos logísticos». Zaragoza, 2007.

[13] L. Mora, «Los indicadores claves del desempeño logístico, de Indicadores de la Gestión Logística».

[14] FEDEGAN, «Normatividad Fedegan».

[15] INVIMA, «Normatividad INVIMA».

[16] M. Olaya, M., «Logística Integral» 2009. 
